Revue internationale P.M.E.

Économie et gestion de la petite et moyenne entreprise

Revure

internationale

PME

\title{
L'investissement direct à l'étranger de la petite et moyenne entreprise : la PME multinationale
}

\section{Enrique Claver Cortès et Diego Quer Ramón}

Volume 16, numéro 1, 2003

URI : https://id.erudit.org/iderudit/1008434ar

DOI : https://doi.org/10.7202/1008434ar

Aller au sommaire du numéro

Éditeur(s)

Presses de l’Université du Québec

ISSN

0776-5436 (imprimé)

1918-9699 (numérique)

Découvrir la revue

Citer cette note

Claver Cortès, E. \& Quer Ramón, D. (2003). L’investissement direct à l'étranger de la petite et moyenne entreprise : la PME multinationale. Revue internationale P.M.E., 16(1), 105-132. https://doi.org/10.7202/1008434ar

\section{Résumé de l'article}

La réalité économique actuelle est caractérisée par deux phénomènes d'une importance indubitable : la globalisation et le poids significatif des petites et moyennes entreprises (PME) dans les économies. Une modernisation graduelle, la coopération entre elles ou l'entrée sur un nouveau marché comme celui des pays moins développés sont quelques-unes des possibilités auxquelles la PME peut avoir recours pour faire face au défi de la globalisation en utilisant les mêmes armes que l'entreprise multinationale. Elle peut également recourir à une arme traditionnellement utilisée par ces dernières : l'investissement direct à l'étranger (IDE). Le recours à ces différentes possibilités par les PME est l'objet de cette étude. Cette analyse repose sur une enquête menée auprès d'un groupe d'entreprises de moins de 500 employés qui ont utilisé un IDE. 


\title{
L'investissement direct à l'étranger de la petite et moyenne entreprise: la PME multinationale
}

Enrique CLAVER CORTÈS

Diego QUER RAMÓN

Université d'Alicante

\author{
MOTS CLÉS
}

\section{Investissement direct à l'étranger - Petite et moyenne entreprise Facteurs déterminants de l'internationalisation}

\begin{abstract}
RÉSUMÉ
La réalité économique actuelle est caractérisée par deux phénomènes d'une importance indubitable: la globalisation et le poids significatif des petites et moyennes entreprises (PME) dans les économies. Une modernisation graduelle, la coopération entre elles ou l'entrée sur un nouveau marché comme celui des pays moins développés sont quelques-unes des possibilités auxquelles la PME peut avoir recours pour faire face au défi de la globalisation en utilisant les mêmes
\end{abstract}

\section{LES AUTEURS}

EnRIQue Claver Cortés est docteur en sciences économiques et de l'entreprise. Il est professeur d'université et directeur de l'unité de recherche "Organisation des entreprises 》 de l'Université d'Alicante (Espagne). Ses intérêts de recherche sont la direction stratégique, l'internationalisation de l'entreprise et la direction des ressources humaines. Adresse : Département d'organisation des entreprises, Université d'Alicante, Campus de San Vicente del Raspeig, Ap. 99 E-03080, Alicante, Espagne. Courriel : Enrique.claver@ua.es

DIEGO QUER RAMÓN est docteur en sciences de gestion de l'entreprise. II est maître de conférences à l'Université d'Alicante (Espagne). Son activité de recherche vise l'étude de la direction stratégique de l'internationalisation de l'entreprise et de l'investissement direct à l'étranger comme stratégie d'entrée. Adresse : Département d'organisation des entreprises, Université d'Alicante, Campus de San Vicente del Raspeig, Ap. 99 E-03080, Alicante, Espagne. Courriel : diego.quer@ua.es 
armes que l'entreprise multinationale. Elle peut également recourir à une arme traditionnellement utilisée par ces dernières: l'investissement direct à l'étranger (IDE). Le recours à ces différentes possibilités par les PME est l'objet de cette étude. Cette analyse repose sur une enquête menée auprès d'un groupe d'entreprises de moins de 500 employés qui ont utilisé un IDE.

\section{ABSTRACT}

The present economic reality is characterised by two undoubtedly relevant phenomenas : globalisation and the important weight of small - and medium-sized enterprises (SME's). The monitoring of a modernisation process, the inter-enterprise co-operation or the consideration of a less developed country as a business target are some of the factors that can make it possible for an SME to respond to the challenge of globalisation with an arm that is traditionally considered only within reach of the large multinational enterprise: foreign direct investment (FDI). The assessment on the importance of these determining factors along with an approach to the internationalisation process displayed by less significant enterprises will constitute the central axis of this research work, the empirical contribution of which has focused on a group of enterprises with less than 500 employees which count on some FDl's.

\section{RESUMEN}

La realidad económica actual está caracterizada por dos fenómenos de indudable relevancia: la globalización y el importante peso de las pequeñas y medianas empresas (PyMEs) en el tejido empresarial. El seguimiento de un proceso gradual, la cooperación interempresarial o la consideración de un país menos desarrollado como destino, son algunos de los factores que pueden posibilitar que la PyME responda al reto de la globalización con un arma tradicionalmente considerada sólo al alcance de la gran empresa multinacional : la inversión directa en el exterior (IDE). La valoración de la importancia de estos factores determinantes junto con una aproximación al proceso de internacionalización desplegado por las empresas de menor dimensión es el eje central de este trabajo, cuya aportación empírica se ha centrado en un colectivo de empresas con menos de 500 empleados que cuentan con alguna IDE.

\section{ZUSAMMENFASSUNG}

Die aktuelle wirtschaftliche Realität ist charakterisiert durch zwei Phänomene von einer unzweifelhaften Bedeutung : die Globalisierung und das signifikante Gewicht der kleinen und mittleren Unternehmen in der Wirtschaft. Die fortschreitende, schrittweise Modernisierung, die Zusammenarbeit zwischen den Betrieben oder der Eintritt in neue Märkte wie diejenigen der weniger entwickelten Länder sind einige der Zufluchtsmöglichkeiten der KMU, der Herausforderung der Globalisierung gegenüberzutreten, indem die Waffen der multinationalen Unternehmen angewendet werden: Die KMU können wie die multinationalen Unternehmen die traditionell genutzte Waffe der direkten Investitionen im Ausland nutzen. Die Zuflucht der kleinen und mittleren Unternehmen zu den verschiedenen Möglichkeiten ist

Revue internationale P.M.E., vol. 16, nº 1, 2003 
Gegenstand dieser Studie. Diese Analyse stützt sich auf eine vergleichende Untersuchung einer Unternehmensgruppe mit weniger als 500 Beschäftigten, die eine Direktinvestition genutzt haben.

\section{Introduction}

Les petites et moyennes entreprises (PME), qui constituent l'un des éléments de base du système économique actuel, ne peuvent échapper au phénomène de la globalisation. C'est en tenant compte de ce dernier que l'on peut défendre avec insistance une stratégie qui non seulement peut aider la PME à maintenir sa compétitivité, mais qui contribue également à son développement, soit l'internationalisation. Mais la PME, en plus de considérer du point de vue stratégique le marché international, peut aussi atteindre un plus haut degré d'intensité dans le processus d'internationalisation jusqu'au point d'effectuer des investissements directs à l'étranger (IDE) et d'acquérir ainsi un caractère multinational. Afin de franchir ce saut qualitatif dans son processus d'internationalisation, toute une série de facteurs déterminants qui atténuent les nombreux risques que comporte cette modalité d'entrée devront favoriser la PME. Traditionnellement, l'IDE a été associé à de grandes entreprises par des filiales implantées dans de nombreux pays ; mais ces derniers temps, il a commencé à s'ouvrir à la PME pour être analysé par les chercheurs.

C'est dans ce contexte que nous devons rappeler l'existence de trois façons de pénétrer dans un pays étranger. La première façon est l'exportation, caractérisée fondamentalement par une production centrée dans le pays d'origine à partir duquel se réalise le service sur les marchés internationaux moyennant des transactions commerciales. Le deuxième groupe de stratégies d'internationalisation est constitué par les accords contractuels dont l'aspect le plus important est la coopération avec des entreprises du pays destinataire. Finalement, la troisième formule d'entrée est l'IDE. À la différence des deux façons précédentes, cette dernière exige un apport de capital de la part de l'entreprise dans le pays de destination. Par conséquent, cette dernière présente les plus hauts niveaux d'engagement de ressources, de risques, de bénéfices potentiels et de contrôle sur les opérations extérieures. De plus, à partir du moment où l'entreprise adopte l'IDE comme stratégie d'entrée, elle devient multinationale; c'est-à-dire qu'elle met sur pied une installation permanente à caractère productif ou commercial dans le pays d'accueil.

À partir de cette problématique, cette étude a pour but de réaliser une analyse empirique supplémentaire sur cette relation entre l'IDE et la PME à travers un ensemble d'entreprises de moins de 500 employés qui ont un établissement permanent à l'étranger et qui, par conséquent, peuvent être considérées comme des multinationales.

Revue internationale P.M.E., vol. 16, nº 1, 2003 
Pour cela, nous avons structuré notre travail en trois parties. La première partie est destinée à justifier cette relation d'un point de vue théorique, à partir d'un ensemble de facteurs qui peuvent permettre aux processus d'internationalisation de la PME d'aller au-delà de l'exportation. Dans la deuxième partie, nous developpons la méthodologie suivie et présentons les résultats obtenus. Dans la troisième, nous dégageons une synthèse de ces résultats.

\section{L'investissement direct à l'étranger et la PME : une justification théorique}

L'environnement actuel est caractérisé par deux phénomènes fondamentaux : l'importance des PME dans les économies et la globalisation. Ce dernier processus se traduit par une série d'opportunités et de menaces pour la PME. Il permet l'apparition de marchés potentiels émergents qui n'étaient pas viables dans le passé et provoque l'arrivée de nouveaux concurrents qui s'appuient, dans de nombreux cas, sur des localisations où le coût de production est plus économique. Dans ce contexte, nous insistons sur le besoin de la PME de s'internationaliser. Comme l'affirment Coviello et McAuley (1999), «il y a eu un changement, qui part de l'intérêt envers l'exportation en elle-même vers la compréhension des processus et des patterns qui expliquent la façon dont les petites entreprises augmentent leur engagement international à travers le temps ».

Au moment d'aborder l'incursion à l'étranger, l'alternative de l'exportation directe suppose moins d'engagement au plan des ressources et moins de risques que d'autres stratégies. C'est pour cela que nous pouvons la considérer comme adéquate pour les plus petites entreprises. Dans ce sens, en accord avec Buckley (1989), nous trouvons deux types de limites à ce que la PME effectue plutôt un IDE: les limites internes (pénurie de capitaux, réticence à acquérir des ressources externes par crainte de perdre le contrôle, manque d'habiletés et de temps, existence de problèmes d'information, etc.) et celles externes (dérives du marché, danger d'une possible acquisition et restrictions institutionnelles). Cependant, comme nous l'avons déjà signalé, la globalisation présente une série de défis plus grands que celui de la simple exportation : le fait que la PME regarde à l'international avec un horizon temporel à long terme. Choisir un plus grand potentiel de bénéfices, profiter des avantages de localisation, éviter les barrières à l'exportation, etc., ne sont que quelques avantages pour investir à l'étranger, face à d'autres modalités d'internationalisation moins ambitieuses. Dans ce contexte et au-delà de l'opinion de Mahone et de Choudhury (1995), qui affirment que « les petites et moyennes entreprises peuvent jouer un rôle important face au défi de la globalisation avec une croissance de leurs exportations », nous pouvons dire plutôt, comme le fait Cazorla (1996), que «l'investissement direct constitue un choix stratégique à portée de la petite et moyenne entreprise ». Pour que cela soit possible, il faut réunir une série de facteurs que nous détaillerons plus bas.

Revue internationale P.M.E., vol. 16, nº 1, 2003 


\subsection{Les théories de I'IDE}

L'un des courants traditionnels des analyses favorisant le recours à l'IDE comme modalité d'incursion à l'international s'appuie sur le phénomène de l'entreprise multinationale $(\mathrm{EM})^{1}$. C'est dans ce sens que l'on peut relever les trois approches suivantes : la théorie de l'existence d'avantages propres, dont les deux représentants les plus importants sont Hymer (1976) et Kindleberger (1969); la théorie d'internalisation, introduite par Buckley et Casson (1976) et par Rugman (1981) et la théorie de la localisation, où l'on retrouve le modèle du cycle de vie du produit sur le marché et l'investissement international, dont les plus grands défenseurs sont Vernon (1966, 1973 et 1979) et Wells (1968).

L'intégration de ces trois théories précédentes est faite dans le fameux paradigme éclectique de Dunning (1979, 1981 et 1988). Ce dernier relève le caractère incomplet de ces théories, considérées individuellement. À partir de ces contributions, sa principale hypothèse est que pour que l'entreprise s'engage dans l'IDE, elle doit remplir trois conditions : 1) la possession d'un avantage propre face aux concurrents d'autres nationalités, 2) l'exploitation de cet avantage de façon interne et 3) le recours à l'ensemble de ces avantages avec des facteurs de production en dehors du pays d'origine. Ces trois types d'avantages constituent, en définitive, les conditions nécessaires et suffisantes pour permettre l'IDE. Comme l'indique Dunning (1981), «Les avantages propres répondent au "pourquoi" de l'activité de l'EM; les avantages d'internalisation répondent au "comment" s'effectue l'engagement et ceux de la localisation répondent au "où" s'effectuera la production ".

Bien que, traditionnellement, toutes ces théories de l'IDE aient été appliquées aux grandes entreprises, elles possèdent également une valeur explicative pour la PME, comme nous le verrons plus loin. Cependant, il est possible d'isoler une série de facteurs qui peuvent influencer de façon plus directe la décision d'investir à l'étranger.

\subsection{La PME et l'investissement direct à l'étranger : les déterminants spécifiques}

\subsubsection{Le suivi d'un processus graduel}

Selon Alonso (1994), les théories de l'IDE malgré leurs limites sont particulièrement aptes à expliquer le comportement international des grandes entreprises en insistant sur leurs avantages spécifiques et leur capacité de gérer et organiser internement

1. Pour une recension récente des principales théories à ce propos, voir le travail de Pla et Suárez (2000).

Revue internationale P.M.E., vol. 16, nº 1, 2003 
les transactions (internalisation). D'où l'interêt des différentes aproximations adoptées par les modèles du processus d'internationalisation. Ces approches analysent l'internationalisation à partir d'une perspective plus dynamique, en concevant le phénomène comme un processus évolutif et cumulatif à travers lequel l'entreprise acquiert un plus haut degré d'engagement international, selon le niveau d'expérience et de connaissance accumulé et les ressources engagées. En accord avec Andersen (1993), il est possible de différencier deux processus : le modèle d'Uppsala et les modèles qui entrevoient l'internationalisation comme une innovation pour l'entreprise. Les deux cas présentent des aspects communs, comme l'affirment Eriksson et al., (1997). Tous deux se basent sur la théorie du comportement de l'entreprise et présentent l'expérience comme étant un facteur d'une grande importance dans le processus d'internationalisation.

En ce qui concerne le modèle d'Uppsala, nous signalons particulièrement les travaux de Johanson et Wiedersheim-Paul (1975) et de Johanson et Valhne (1977, 1990), qui considèrent que de nombreuses entreprises amorcent leurs activités internationales, quand elles sont relativement petites, en développant graduellement leurs opérations étrangères en passant par ce que nous appelons un cheminement graduel : 1) des exportation non régulières, 2) des exportations à travers des représentants indépendants ou agents, 3) des exportations directes et, enfin, 4) des productions spécialisées pour l'exportation. Cette évolution est influencée par deux éléments d'une grande importance. D'une part, la distance psychologique (différences du point de vue de la langue, de la culture, des systèmes politiques, du niveau d'éducation, du niveau de développement industriel, etc.), distance que l'on évitera a priori et, d'autre part, le volume du marché potentiel. Dans ce cas, l'entreprise donnera préférence aux opérations sur des petits marchés où un engagement initial de ressources moins important est exigé et où il existe des industries nationales moins compétitives.

Le deuxième groupe de théories du processus d'internationalisation (Andersen, 1993) porte sur la «séquence d'apprentissage en relation avec l'adoption d'une innovation ». En effet, le processus d'internationalisation possède un caractère innovateur, car il comporte pour l'entreprise un changement dans sa structure d'organisation, dans ses objectifs stratégiques, dans son programme de marketing et, parfois, dans ses conditions préalables de production (Alonzo, 1993). Dans ce contexte, nous trouvons une série de contributions différentes qui définissent une série d'étapes du processus ${ }^{2}$. La nuance qui le différencie du modèle d'Uppsala concerne l'accent mis sur les caractéristiques de l'entreprise qui entreprend l'internationalisation.

2 Les principaux travaux dans cette ligne de pensée sont ceux de Bilkey et Tesar (1977), Cavusgil (1980), Czinkota (1982), Reid (1981) et Root (1982). 
Diverses études empiriques ont confirmé la validité de ces approches pour expliquer l'internationalisation des PME. Cependant, elles donnent aussi un aperçu de l'applicabilité des théories de l'IDE exposées auparavant. C'est ainsi qu'en employant la méthodologie de l'étude des cas, Martínez Mora (1997) explique que les théories de l'IDE et l'approche graduelle peuvent s'appliquer aux entreprises plus petites. Pour sa part, Cazorla (1996, 1997), à l'aide d'une enquête sur un échantillon plus grand d'entreprises, a relevé trois facteurs qui déterminent la stratégie d'investissement des PME: les facteurs de nature commerciale (augmenter ou consolider les exportations, que ce soit dans le pays d'accueil ou en utilisant ce dernier comme plate-forme d'exportation), l'exploitation des avantages propres (profiter d'une technologie supérieure à celle des concurrents nationaux du pays développé) et des facteurs de localisation (fondamentalement le coût de la maind'œuvre). Par conséquent, les éléments de la théorie de l'existence des avantages propres et de la théorie de la localisation sont sous-jacents. Deux autres conclusions supplémentaires intéressantes de ce travail peuvent être trouvées. D'une part, il s'agit en grande partie d'entreprises qui ont basé leur succès international sur un grand nombre d'années d'expérience sur le marché national. D'autre part, bon nombre d'entre elles ont acquis leur dimension internationale de façon graduelle, c'est-à-dire en adoptant avec le temps et en fonction de l'expérience accumulée des formules institutionnelles chaque fois plus complexes, mais sans suivre successivement les différentes étapes suggérées par le modèle d'Uppsala.

Enfin, Coviello et McAuley (1999), dans la recension des recherches empiriques sur l'internationalisation de la PME, relèvent trois courants qui doivent être pris en compte de façon complémentaire. Ainsi, l'entreprise peut chercher l'internalisation des activités au moment de choisir des marchés et des stratégies d'entrée (théories de l'IDE); parallèlement, elle peut internationaliser de façon croissante ses opérations tout en suivant une série d'étapes (modèles du processus); et, en plus, elle peut faire des efforts d'externalisation de certaines activités à travers des relations avec d'autres entreprises (perspective de réseaux). Cette troisième approche, qui peut être un facteur déterminant et spécifique pour soutenir l'IDE, que nous exposons par la suite, montre qu'une grande partie du succès international de la PME relève de ses relations avec les clients, fournisseurs, concurrents, institutions, etc. ${ }^{3}$.

3. Par rapport à la théorie d'internalisation, Johanson et Mattsson (1988) indiquent que le réseautage favorise l'apprentissage avec d'autres entreprises et donne la possibilité d'externaliser certaines activités. D'autre part, à l'opposé du modèle d'Uppsala, l'approche réticulaire se centre sur les relations avec le marché pour accumuler des connaissances (beaucoup plus que sur le développement interne de l'entreprise); ce modèle est plus approprié dans des situations où le marché et l'entreprise présentent un haut niveau d'internationalisation (puisque, dans ce contexte, il ne requiert pas une implication aussi grande que pour d'autres changements stratégiques).

Revue internationale P.M.E., vol. 16, nº 1, 2003 


\subsubsection{Activité conjointe avec d'autres entreprises}

Le deuxième facteur déterminant touche au recours à la coopération entre entreprises au moment d'aborder les processus d'internationalisation. En effet, selon Rialp et Rialp (1996) et Darréon et Faiçal (1993), l'apprentissage obligé associé à l'internationalisation, compte tenu du manque de ressources de nombreuses entreprises pour développer les nouvelles connaissances requises seules, transforme les accords en mécanismes de plus en plus recherchés pour entrer sur de nouveaux marchés internationaux. Cela peut favoriser l'IDE puisque, comme l'affirme Camisón (1997), «La coopération peut se voir comme une option stratégique qui permet aux organisations de dimensions réduites d'avoir accès aux ressources financières, techniques et humaines - nécessaires pour instaurer et soutenir des infrastructures commerciales qui puissent faciliter le développement vers un stade plus avancé dans leur processus d'internationalisation». Cet auteur distingue deux formes typiques de coopération internationale: les entreprises conjointes internationales (formées par un partenaire étranger et une entreprise locale, où le premier cherche l'accès à une capacité locale) et la coopération à caractère national (qui permet aux entreprises de petite taille d'avoir accès, conjointement, à certains pays où l'accès individuel est très compliqué à cause des difficultés ou du volume d'investissement requis) ${ }^{4}$.

Mais l'accès aux capacités locales ou la possibilité de se lier à des capacités étrangères ne constituent pas les seuls avantages que présente la coopération internationale. Ces accords peuvent permettre une accumulation de connaissances grâce à laquelle l'entreprise pourra éviter certaines étapes proposées par l'approche graduelle et s'internationaliser même dès sa création. Cela nous amène à ce que Oviatt et McDougall (1994) ont appelé la nouvelle entreprise internationale (NEI) pour faire référence à «une organisation qui, dès le début, cherche à obtenir un avantage compétitif significatif à partir du recours à diverses ressources pour obtenir certaines économies d'échelle ou de champ ${ }^{5}$. De ce point de vue, selon Iborra et Ripollés (1997) et Hitt et Bartkus (1997), nous pouvons affirmer que les réseaux

4. Dans ce sens-là, face aux travaux traditionnels portant exclusivement sur les coentreprises où participent un membre local et un membre étranger, on peut se référer aux études de Pan et Tse (1996), qui se centrent sur les stratégies de coopération entre deux entreprises étrangères sur le marché extérieur et qui signalent que plus les risques et les coûts d'opérer individuellement augmentent, plus le besoin de coopérer se rélève nécessaire.

5. McDougall, Shane et Oviatt (1994) expliquent que les théories d'internationalisation ne sont pas exactes au moment d'expliquer le processus de formation des NEI pour deux raisons: elles présument que l'entreprise devient internationale après s'être formée et elles se centrent sur l'entreprise en ignorant le rôle de l'individu, c'est-à-dire de l'entrepreneur et de son réseau.

Revue internationale P.M.E., vol. 16, nº 1, 2003

(C) 2003 - Presses de l'Université du Québec

Édifice Le Delta I, 2875, boul. Laurier, bureau 450, Sainte-Foy, Québec G1V 2M2 • Tél.: (418) 657-4399 - www.puq.uquebec.ca

Tiré de: Revue internationale P.M.E., vol. 16, n 1 , sous la direction de Pierre-André Julien. 
formels et informels dont font partie les NEI et, plus spécialement, leur chef d'entreprise, vont leur fournir les ressources tangibles et intangibles nécessaires pour réussir rapidement leur internationalisation et diriger le processus d'une façon plus efficace ${ }^{6}$. Cependant, comme le signalent Chetty et Blankenburg Holm (2000), la direction de la PME peut ne pas être préparée pour se ranger à l'idée d'internationalisation qui émane de ces réseaux. Cela nous amène au facteur déterminant suivant.

\subsubsection{Une mentalité orientée vers l'internationale}

L'orientation internationale est considérée par Veciana et Genescá (1994) comme une des décisions fondamentales appartenant à la direction et permettant d'augmenter la concurrence entre entreprises, à partir de l'argument que cette décision «doit dépasser l'analyse proprement commerciale ou productive et acquérir un caractère beaucoup plus général touchant toute la culture de l'organisation. Elle doit être attentive aux changements technologiques, aux nouvelles orientations de la gestion, aux procédés et aux démarches qui devront être suivies en dehors des frontières, car cela constitue une source inépuisable d'idées, d'innovations et d'adaptations qui ont été déjà expérimentées de manière empirique».

Selon Reid (1981), il est probable que le comportement d'exportation soit plus influencé par les décisions individuelles dans la petite entreprise et beaucoup moins soumis aux relations structurelles typiques de la grande entreprise. Reuber et Fischer (1997) acceptent cette idée quand ils affirment que tandis que dans les grandes entreprises la disponibilité de ressources ou l'internalisation des marchés peuvent expliquer l'internationalisation, dans les petites ou les nouvelles entreprises (ayant habituellement moins de ressources), les habiletés et les connaissances de la haute direction influencent beaucoup plus la démarche d'internationalisation. D'où l'importance du rôle joué par le fondateur et le nombre de déplacements qu'il a réalisés à l'étranger, les langues qu'il parle, son lieu de naissance, ou son expérience à l'étranger ${ }^{7}$. Quant à Suárez, García et Álamo (1999), lors de l'examen

6. Ces réseaux peuvent faire référence à des organisations (concurrents, clients, fournisseurs, institutions...) ou à des personnes (réseaux sociaux ou informels). Ces derniers sont définis ainsi par Planellas (1997): «il s'agit du réseau de relations établis par le fondateur de l'entreprise avec d'autres personnes externes à l'entreprise, mais qui font partie de son environnement proche. L'objectif de construire ce réseau est la recherche hors de l'entreprise des ressources que le fondateur de l'entreprise a besoin et dont il ne dispose pas à l'interne: information, technologie, conseil, financement, etc.».

7. Dans ce sens, deux recensions des principales études empiriques effectuées sur le comportement exportateur de la PME, réalisées par Miesenbock (1988) et Gibiat (1994), constatent que le responsable de la prise de décision constitue la variable essentielle expliquant la démarche.

Revue internationale P.M.E., vol. 16, nº 1, 2003

(C) 2003 - Presses de l'Université du Québec

Édifice Le Delta I, 2875, boul. Laurier, bureau 450, Sainte-Foy, Québec G1V 2M2 • Tél.: (418) 657-4399 - www.puq.uquebec.ca

Tiré de: Revue internationale P.M.E., vol. 16, n 1, sous la direction de Pierre-André Julien. 
des facteurs déterminants internes d'un plus grand engagement international de la PME, ceux-ci ont vérifié également de façon empirique le rôle crucial que joue la variable direction. Plus concrètement, ils élaborent un modèle explicatif d'engagement à l'exportation dans lequel sont incluses deux catégories en relation avec la direction de l'entreprise ${ }^{8}$ : les caractéristiques de la direction (parmi lesquelles son niveau d'études et sa formation à l'étranger ont une influence positive très importante) et ses perceptions (où la croyance que l'internationalisation améliorera la capacité concurrentielle de l'entreprise et aura une influence positive sur ses résultats, alors que la perception d'obstacles de connaissance aura une influence négative). Le travail de Valenzuela (2000) permet aussi de soutenir cette analyse en montrant que le niveau d'internationalisation de l'entreprise est déterminé par l'accumulation de ressources nécessaires pour la création de capacités exportatrices, spécialement la capacité du responsable à prendre des décisions pour accepter des niveaux d'internationalisation croissants.

En dépit du fait que tous ces auteurs parlent de «comportement à l'exportation», nous considérons que nous pouvons parler de « comportement international », car les idées qu'ils exposent peuvent être extrapolées à d'autres stratégies comme l'IDE. Il est même probable que, dans ce cas, l'importance de la direction soit encore plus forte.

\subsubsection{Les conditions préalables}

D'un autre point de vue, Korhonen, Luostarinen et Welch (1996) relèvent un autre facteur, soit d'avoir effectué préalablement des opérations internationales à l'intérieur du pays d'accueil, comme, par exemple, des opérations de production (importations de machines, de matières premières, de composants, etc.), des opérations de service (montages, services d'entretien) et d'opérations touchant le savoir (licences, franchises, etc.).

Il est aussi très important d'avoir eu des activités d'importation; cela a pu favoriser des voyages à l'étranger, des recherches sur les prix et sur des fournisseurs, des négociations avec ces derniers, etc., en définitive, toutes expériences dans le domaine international pouvant être utilisées au moment d'effectuer des activités de vente à l'extérieur, en diminuant les obstacles et l'incertitude de ces situations. Dans le cas de la PME où la même personne peut prendre en charge les importations et les exportations, la situation favorisera cette liaison. Comme dans le cas précédent, nous considérons que ces idées sont transférables au cas de l'IDE.

8. Le modèle se complète avec une troisième catégorie qui inclut certaines caractéristiques de l'entreprise: grand volume, importance du niveau d'expansion nationale, supériorité dans le développement de nouveaux produits et haut degré d'intégration verticale. 


\subsubsection{La destination dans des pays en voie de développement (PVD)}

Un autre élément très important est l'influence des facteurs de localisation liés au pays de destination. Selon Giddy et Young (1982), les avantages monopolistiques des PME seront relativement faibles ou provisoires; c'est la raison pour laquelle elles devront faire face à de plus grandes barrières à l'entrée au moment d'investir, barrières qui devront être compensées par des facteurs spécifiques de localisation comme, par exemple, le recours à des associés locaux qui apporteront des connaissances et du capital; les concurrents principaux seront des entreprises locales; l'existence de marchés garantis (p. ex., en agissant déjà comme sous-traitant de grandes entreprises); des primes fiscales ou financières qui favorisent les investisseurs étrangers ; une proximité linguistique, culturelle ou physique avec le pays d'accueil et un manque de compétitivité de la part des concurrents du pays d'accueil, ce qui arrive habituellement dans des pays moins développés économiquement que le pays d'origine.

Ce dernier facteur a une importance particulière, car ces pays sont considérés comme des marchés de plus en plus intéressants. De fait, selon Arnold et Quelch (1998), un changement de dénomination est en train de se produire pour désigner cette nouvelle façon de les voir: l'expression «marchés émergents» a remplacé les expressions de «pays moins développés », «pays en voie d'industrialisation » ou même «pays du tiers monde », qui les faisaient apparaître comme des sources bon marché de matières premières et de main-d'œuvre au lieu de marchés potentiels. L'IDE dans ces pays présente des avantages réciproques, d'après le Conseil supérieur de chambres de commerce, d'industrie et de navigation (1990) ou encore d'après Donckels et Lambrecht (1995). Pour les entreprises qui veulent investir (spécialement pour les PME), ces pays leur offrent la possibilité de s'internationaliser; pour les pays d'accueil, l'investissement étranger améliorera leur balance des paiements, facilitera l'accès à de nouvelles technologies et permettra d'augmenter leurs exportations 9 .

Les résultats obtenus dans le travail de Cazorla (1996) soutiennent également cette hypothèse. D'une part, cet auteur montre que l'IDE des PME espagnoles dans les PVD se limite généralement à l'assistance technique et à l'apport de biens

9. Ce facteur est sous-jacent dans la reformulation du modèle du cycle du produit de Vernon (1979) et il est aussi inclus dans le travail de Martínez Mora (1997). Dans cette dernière étude, on montre que l'Espagne a un niveau de développement supérieur à certains PVD, comme les pays sud-américains, ceux de l'Afrique du Nord ou de l'Europe de l'Est et d'Asie, en dépit d'un développement inférieur par rapport au reste des pays de l'Union européenne ou des É.-U. C'est vers ces pays que se dirigent les IDE réalisés par les entreprises étudiées, car les coûts de main-d'œuvre et une importante demande potentielle ou même la possibilité de les utiliser comme plate-formes d'exportation sont des avantages importants de ces pays.

Revue internationale P.M.E., vol. 16, nº 1, 2003

(C) 2003 - Presses de l'Université du Québec

Édifice Le Delta I, 2875, boul. Laurier, bureau 450, Sainte-Foy, Québec G1V 2M2 • Tél. : (418) 657-4399 - www.puq.uquebec.ca

Tiré de : Revue internationale P.M.E., vol. $16, \mathrm{n}^{\circ} 1$, sous la direction de Pierre-André Julien. 
d'équipement, ce qui donne une idée de l'importance stratégique de ces destinations compte tenu du niveau technologique moyen des entreprises espagnoles. D'autre part, les entreprises qui investissent reçoivent comme principaux bénéfices la création d'emploi et le transfert de nouvelles technologies et de techniques de gestion d'entreprise ${ }^{10}$, et ce indépendamment du niveau de contribution de leurs projets au développement de ces pays.

\subsubsection{L'effet d'entraînement des grandes entreprises}

Un autre élément qui peut favoriser la localisation extérieure des activités d'une PME est l'effet d'entraînement de l'internationalisation préalable d'une grande entreprise avec qui on fait affaire dans le pays d'origine. Dans le processus d'internationalisation, il est plus facile de suivre une entreprise cliente pour laquelle la PME constitue le principal fournisseur (ce qu'on retrouve habituellement dans le secteur de l'automobile) et dont l'image de marque des produits des grandes entreprises est bien vue dans le pays d'accueil (c'est le cas des investissements d'entreprises emblématiques espagnoles en Amérique latine).

\subsubsection{Le soutien public}

Compte tenu des caractéristiques particulières des PME, le rôle des programmes de soutien à l'IDE peut avoir une importance vitale. Dans ce contexte, Alonso et Donoso (1996) soulignent l'importance de soutenir l'engagement à l'international des PME. S'il est important d'encourager aussi l'internationalisation de la PME par l'IDE, les stratégies de soutien doivent adopter des mesures particulières, non seulement pour stimuler l'internationalisation du point de vue commercial, mais aussi du point de vue des activités de production. Pour ces dernières, nous pouvons noter les aides pour s'établir à l'étranger, de façon individuelle ou conjointe, les mesures fiscales qui éliminent les obstacles à l'IDE et qui tentent de compléter l'investissement initial, l'assistance et l'information sur les destinations potentielles; les programmes de formation qui préparent le personnel pour travailler aisément à l'extérieur, etc. ${ }^{11}$.

10. En Espagne, l'importance de ce facteur est corroborée par l'existence de la Compañía Española de Financiación al Desarrollo (COFIDES), qui est une société anonyme de l'Etat à capital mixte qui soutient financièrement des projets d'investissement d'entreprises espagnoles dans les pays moins développés d'Amérique latine, d'Afrique, d'Asie et d'Europe centrale et orientale.

11. En Espagne, à part COFIDES, il convient de souligner le soutien de l'Instituto Español de Comercio Exterior (ICEX) et de l'Instituto de Crédito Oficial (ICO). 
Dans ce contexte, nous pourrions également situer les clubs d'entrepreneurs, les associations patronales et les chambres de commerce. Toutes ces institutions maintiennent habituellement une étroite relation entre elles et constituent des réseaux de coopération qui intègrent aussi les centres de formation comme, par exemple, les universités. La collaboration de l'ensemble de ces institutions peut faciliter une série d'avantages aux PME dans leur aventure internationale.

En définitive, l'ensemble des facteurs mentionnés permet d'établir des liens entre une stratégie d'internationalisation plus ambitieuse (l'IDE) avec la réalité de l'entreprise actuelle (les PME). Autrement dit, ils peuvent justifier un phénomène que certains ont appelé des «multinationales de poche». Le reste de l'exposé aura comme but de démontrer l'évidence empirique de tous ces facteurs.

\section{Objectifs et méthodologie de la recherche}

Le principal objectif de cette recherche empirique est de démontrer que l'IDE est une stratégie d'entrée dans les marchés internationaux à portée de la PME, telle qu'elle est définie par l'Observatoire européen de la PME ${ }^{12}$. La population de l'étude est constituée d'un collectif d'entreprises de moins de 500 employés dont le centre de décision se trouve dans la province d'Alicante (Espagne) et qui comptaient au moins un établissement permanent à l'étranger en 1999.

Notre prétention était d'interviewer personnellement le principal responsable international de chaque entreprise. Pour cela, nous avions besoin d'un répertoire des multinationales établies en Alicante; finalement, nous l'avons élaboré indirectement, car ce recueil était inexistant. Premièrement, nous avons pris contact avec différentes institutions qui pouvaient disposer de données pour notre recherche: Compañía Española de Financiación al Desarrollo (COFIDES), Instituto de Crédito Oficial (ICO), Instituto Valenciano de la Exportación (IVEX) et Instituto de la Mediana y Pequeña Industria Valenciana (IMPIVA) ${ }^{13}$. Deuxièmement, nous nous sommes basés sur deux études récentes sur l'internationalisation de l'entreprise d'Alicante (Calvo, 1997; Martínez Mora, 1997) qui nous ont permis de connaître

12. Divers travaux sur les PME, comme ceux de Hégo (1994), Bayad (1997), Léo (2000) ou Cazorla (1996, 1997), utilisent la classification suivante pour distinguer la taille des entreprises: microentreprises (moins de 10 employés), petites entreprises (entre 10 et 99), entreprises moyennes (entre 100 et 499) et grandes entreprises (à partir de 500 employés).

13. À part ces institutions, nous avons aussi pris contact avec celles-ci : Dirección General de Política Comercial e Inversiones Exteriores, Compañía Española de Seguro de Crédito a la Exportación (CESCE), Instituto Español de Comercio Exterior (ICEX) et Cámara Oficial de Comercio, Industria y Navegación de Alicante. Cependant, les deux premières ont allégué que les noms des entreprises qui investissent étaient confidentiels et les deux dernières manquaient de renseignements. 
certaines entreprises qui avaient déjà investi ou prévoyaient d'investir à court terme à l'étranger. Finalement, nous avons consulté des articles parus récemment: Economía 3 (revue économique sur la province d'Alicante), El Exportador (revue sur le commerce extérieur au plan national), Expansión et Cinco Días (journaux économiques nationaux) et Información (journal à caractère général publié dans la province d'Alicante), pour trouver d'autres entreprises qui auraient investi à l'étranger.

Après avoir éliminé les doublons relevés dans ces sources d'information, nous avons élaboré un premier répertoire ; ce recueil a été peaufiné par des contacts téléphoniques avec les entreprises dont le caractère vraiment investisseur à l'international suscitait des doutes. À la suite de cette étude, nous avons dressé la liste définitive de la population étudiée; soit 41 entreprises de moins de 500 employés. Évidemment, la méthodologie d'élaboration du répertoire, ne nous permet pas d'affirmer sans l'ombre d'un doute qu'il s'agit de la totalité des PME d'Alicante qui investissent à l'étranger. Cependant, nous avons essayé de nous rapprocher le plus possible de ce groupe réel en tenant compte des informations obtenues des sources alternatives mentionnées.

Au lieu de nous limiter à un échantillon, nous avons essayé de joindre toutes les entreprises pointées. Avec cet objectif, nous avons pris contact avec le principal responsable des relations internationales de chaque entreprise pour avoir un entretien personnel structuré par un questionnaire. Trente-deux entreprises ont collaboré, ce qui représente un taux de réponses de $78 \%$. En employant cette méthodologie pour obtenir l'information, il se peut que deux erreurs aient été commises, erreurs que nous avons tenté de contrôler. En premier lieu, l'erreur d'échantillonnage peut provenir du fait que l'enquête n'a pas porté sur la totalité de la population. En ce sens, les modèles mathématiques permettent de quantifier cette erreur, pour une variable déterminée, seulement dans le cas d'un échantillon aléatoire simple. En supposant ce processus d'échantillonnage et en considérant comme variable d'importance la propension à exporter de chaque entreprise (pourcentage d'exportation sur les ventes totales), il serait donc possible de délimiter l'erreur. Ainsi, nous avons obtenu une propension à l'exportation moyenne de $52,5 \%$ et, pour un intervalle de confiance de 95,5\%, l'erreur absolue commise serait de $4,5 \%$.

En second lieu, l'erreur indépendante de l'échantillonnage peut provenir des erreurs commises dans les réponses ou des non-réponses de la part des entreprises. Quant aux erreurs possibles commises par les répondants, nous avons tenté de les réduire de diverses façons : en essayant d'avoir un entretien personnel avec le principal responsable international de chaque entreprise (afin d'éviter le manque de connaissance des sujets traités) et en réalisant un entretien pilote (qui a servi pour retoucher, voire éliminer certaines questions pouvant porter à confusion). Les non-réponses concernent neuf entreprises que nous n'avons pu enquêter. Pour 
découvrir jusqu'à quel point ce biais a pu être significatif, nous avons comparé les entreprises qui ont répondu et celles qui s'en sont abstenues ${ }^{14}$. Nous avons donc réalisé une série d'analyses en utilisant les variables qui permettent de mieux classer les entreprises et qui font référence - en ce qui concerne les entreprises non enquêtées - aux données actualisées dans l'un des répertoires disponibles. De ces analyses, nous pouvons dégager les conclusions suivantes, qui nous permettent de minimiser l'existence d'un biais significatif : d'une part, la distribution sectorielle du collectif qui a répondu est assez semblable à celle de la population, et d'autre part, des différences statistiquement significatives n'ont pas été relevées entre les deux groupes d'entreprises (les répondants et les non-répondants) dans les moyennes des variables suivantes: nombre d'employés, volume absolu d'exportation, propension à l'exportation et nombre d'IDE.

\section{Résultats et discussion}

\subsection{Analyse descriptive des PME étudiées}

Nous commençons l'exposé des résultats de la recherche empirique avec une analyse descriptive du groupe étudié. D'abord, nous constatons que $62,5 \%$ des entreprises se concentrent sur quatre secteurs (tableau 1): chaussures (industrie auxiliaire incluse), jouets, alimentation et textile; cela est représentatif de la réalité des entreprises implantées dans la région géographique choisie, soit la province d'Alicante. Signalons qu'il s'agit des quatre secteurs ayant la plus forte tradition et la plus forte présence à l'échelle internationale. Ensuite, des 29 entreprises $(90,6 \%)$ qui à la fois produisent et commercialisent des produits, deux ont un caractère exclusivement commercial et une entreprise offre des services.

En ce qui concerne la taille des entreprises, nous avons relevé une moyenne de 136 employés et 19,7 millions d'euros de chiffre d'affaires. Dans le tableau 2, nous présentons la distribution de fréquences de la variable utilisée dans ce travail pour délimiter cette taille.

Quant aux variables descriptives portant sur les aspects internationaux, il faut signaler, premièrement, que la plupart des entreprises ont une ancienneté internationale de dix à trente ans - soit le temps écoulé après l'amorce du processus d'internationalisation. La moyenne est de vingt ans et seulement six entreprises ont commencé leur aventure internationale dans la dernière décennie (tableau 3).

14. Cette méthode ainsi que d'autres visant à évaluer le biais dérivé du manque de réponse sont exposées par Armstrong et Overton (1977).

Revue internationale P.M.E., vol. 16, nº 1, 2003 
TABLEAU 1

Distribution des entreprises par secteur

\begin{tabular}{lccc}
\hline & Fréquence & Pourcentage & Pourcentage cumulé \\
\hline Chaussure & 6 & 18,8 & 18,8 \\
Jouets & 5 & 15,6 & 34,4 \\
Alimentation & 4 & 12,5 & 46,9 \\
Textile & 3 & 9,4 & 56,3 \\
Composants en chaussure & 2 & 6,3 & 62,5 \\
Articles de sports & 1 & 3,1 & \\
Climatisation industrielle & 1 & 3,1 & \\
Développement de logiciels & 1 & 3,1 & \\
Pharmaceutique & 1 & 3,1 & \\
Hygiène industrielle & 1 & 3,1 & \\
Pierre naturelle & 1 & 3,1 & \\
Produits en caoutchouc & 1 & 3,1 & \\
Polymère et caoutchouc & 1 & 3,1 & \\
Produits cosmétiques & 1 & 3,1 & \\
Produits chimiques & 1 & 3,1 & \\
Portes & 1 & 3,1 & \\
Traitement des résidus solides urbains & 1 & 3,1 & \\
\hline Total & 32 & 100,0 & \\
\hline
\end{tabular}

TABLeau 2

Distribution des entreprises selon le nombre d'employés

\begin{tabular}{lccc}
\hline & Fréquence & Pourcentage & Pourcentage cumulé \\
\hline Moins de 10 & 2 & 6,3 & 6,3 \\
Entre 10 et 99 & 12 & 37,5 & 43,8 \\
Entre 100 et 249 & 10 & 31,3 & 75,0 \\
Entre 250 et 499 & 8 & 25,0 & 100,0 \\
\hline Total & 32 & 100,0 & \\
\hline
\end{tabular}

TABLEau 3

Distribution des entreprises selon leur ancienneté internationale (par an)

\begin{tabular}{lccc}
\hline & Fréquence & Pourcentage & Pourcentage cumulé \\
\hline Moins de 10 & 6 & 18,8 & 18,8 \\
Entre 10 et 19 & 13 & 40,6 & 59,4 \\
Entre 20 et 29 & 7 & 21,9 & 81,3 \\
Entre 30 et 39 & 3 & 9,4 & 90,6 \\
Entre 40 et 49 & 3 & 9,4 & 100,0 \\
\hline Total & 32 & 100,0 & \\
\hline
\end{tabular}


Deuxièmement, l'engagement exportateur peut être évalué à partir de deux mesures différentes, l'une absolue et l'autre relative. Dans le premier cas, nous avons obtenu un volume moyen absolu d'exportation de 10,5 millions d'euros annuellement et, dans le second cas, une propension moyenne à l'exportation de $52,5 \%$. Comme l'illustre le tableau 4, la plupart destinent aux marchés extérieurs un peu plus de la moitié de leurs ventes totales, ce qui nous amène à penser que les entreprises ont un engagement avec l'IDE issu d'une position exportatrice consolidée ${ }^{15}$.

TABLEAU 4

Distribution des entreprises selon leur propension à l'exportation

\begin{tabular}{lccc}
\hline & Fréquence & Pourcentage & Pourcentage cumulé \\
\hline Moins de $25 \%$ & 5 & 16,1 & 16,1 \\
Entre $25 \%$ et $49 \%$ & 6 & 19,4 & 35,5 \\
Entre $50 \%$ et $74 \%$ & 14 & 45,2 & 80,6 \\
Entre $75 \%$ et $99 \%$ & 6 & 19,4 & 100,0 \\
\hline Total & 31 & 100,0 & \\
\hline
\end{tabular}

Après avoir effectué ce premier rapprochement descriptif du groupe d'entreprises étudiées, nous allons poursuivre notre travail en essayant d'apporter une évidence empirique en ce qui concerne les deux principaux aspects abordés dans la partie théorique, soit les théories touchant l'IDE et les facteurs déterminants propres aux PME

\section{2. Évidence empirique des théories de l'IDE}

Cette analyse vise à vérifier le degré d'influence du paradigme éclectique en ce qui concerne la décision d'investir. Pour cela, nous avons posé une question directe afin d'établir l'importance (sur une échelle de 0 à 10) des trois avantages qui le configurent et qui sont à l'origine d'autres variables, dont les moyennes sont recueillies au tableau 5. D'autre part, nous les avons ensuite classés selon cinq catégories, en montrant la distribution de fréquences au tableau $6^{16}$.

15. Dans le tableau 4, il manque une entreprise qui n'a pas pu nous indiquer sa propension à l'exportation à cause de sa récente internationalisation.

16. Comme nous pouvons le voir, cette analyse ne considère pas les 32 PME étudiées qui investissent. Cela s'explique par l'exclusion d'une entreprise qui a acquis les délégations à l'étranger d'une autre entreprise espagnole absorbée au lieu de prendre la décision d'investir. En plus, l'entreprise de services n'a pas tenu compte du facteur internalisation puisqu'elle n'effectuait pas d'exportations.

Revue internationale P.M.E., vol. 16, nº 1, 2003 
TABLEAU 5

Influence moyenne des avantages définis par le paradigme éclectique

\begin{tabular}{lcc}
\hline & N & Moyenne \\
\hline Influence des avantages propres sur la concurrence (propriété) & 31 & 7,06 \\
Influence des avantages de localisation des marchés (localisation) & 31 & 5,42 \\
Influence des avantages de gérer et d'organiser à l'interne & & \\
les transactions de façon à réduire leurs coûts (internalisation) & 30 & 7,20 \\
\hline
\end{tabular}

TABleau 6

Influence des avantages définis par le paradigme éclectique

\begin{tabular}{lcccrcc}
\hline & $\begin{array}{c}\text { Influence des } \\
\text { avantages } \\
\text { «propriété» }\end{array}$ & $\begin{array}{c}\text { Influence des } \\
\text { avantages } \\
\text { «localisation» }\end{array}$ & $\begin{array}{c}\text { Influence des avantages } \\
\text { «internalisation» }\end{array}$ \\
\cline { 2 - 6 } & Nombre & $\%$ & Nombre & $\%$ & Nombre & $\%$ \\
\hline Rien & 2 & 6,5 & 9 & 29,0 & 4 & 13,3 \\
Peu & 2 & 6,5 & 1 & 3,2 & 0 & 0,0 \\
Modérément & 5 & 16,1 & 4 & 12,9 & 3 & 10,0 \\
Assez & 15 & 48,4 & 11 & 35,5 & 15 & 50,0 \\
Beaucoup & 7 & 22,6 & 6 & 19,4 & 8 & 26,7 \\
\hline Total & 31 & 100,0 & 31 & 100,0 & 30 & 100,0 \\
\hline
\end{tabular}

Ces deux analyses nous ont permis de relever que les avantages touchant la propriété (les avantages distinguant l'entreprise) et l'internalisation (le fait de gérer et d'organiser à l'interne les transactions de façon à réduire les coûts) ont été considérés comme plus influents que ceux liés à la localisation (l'ouverture de nouveaux marchés à l'étranger). De fait, les moyennes des deux premières variables se situent au-dessus de 7 (sur une échelle de 0 à 10 ), tandis que la moyenne de la troisième variable dépasse à peine 5 . En distinguant par catégorie, un peu plus de $70 \%$ des entreprises considère que les avantages compétitifs et d'internalisation ont eu assez ou beaucoup d'influence au moment d'investir, alors que 32,2\% attribue une importance nulle ou très faible au facteur de localisation. Ce résultat doit être analysé avec soin et considéré uniquement comme une perception de la direction lorqu'on mesure l'influence des avantages du paradigme éclectique. En fait, pour évaluer avec justesse l'importance de chacune de ces variables, il aurait fallu tenir compte de l'objectif concret de chaque IDE: recherche de ressources complémentaires ou d'actifs stratégiques, élargissement de marchés, etc. 


\subsection{Déterminants spécifiques de l'IDE effectué par les PME}

Comme nous l'avons déjà relevé dans la partie théorique, les modèles du processus graduel d'internationalisation ont une valeur explicative particulière dans le cas de la PME. C'est pourquoi nous allons examiner en détail le caractère graduel de l'internationalisation que défendent ces approches, pour ensuite étudier le reste des déterminants spécifiques. Premièrement, le tableau 7 montre que la plupart des entreprises $(59,4 \%)$ contrôlent un seul IDE; cinq entreprises seulement possèdent au moins cinq établissements permanents à l'étranger. Cela signifie qu'il s'agit d'entreprises ayant une présence internationale encore relativement faible, ce qui nous amène à les qualifier de multinationales naissantes.

TABLEAU 7

Distribution des entreprises selon le nombre d'IDE

\begin{tabular}{lccc}
\hline & Fréquence & Pourcentage & Pourcentage cumulé \\
\hline 1 IDE & 19 & 59,4 & 59,4 \\
2 IDE & 5 & 15,6 & 75,0 \\
3 IDE & 3 & 9,4 & 84,4 \\
5 IDE & 3 & 9,4 & 93,8 \\
IDE & 1 & 3,1 & 96,9 \\
10 IDE & 1 & 3,1 & 100,0 \\
\hline Total & 32 & 100,0 & \\
\hline
\end{tabular}

Sur le plan géographique, le tableau 8 montre que plus de la moitié des 69 IDE relevés (soit 55,1\%) se concentrent dans six pays : les États-Unis (10), l'Allemagne (8), le Portugal (6), le Brésil (5), le Mexique (5) et la France (4). En les regroupant par région, on trouve la distribution suivante : l'Union européenne (26), le reste de l'Europe (5), le Mercosur (8), le reste de l'Amérique latine (11), les États-Unis (10), l'Asie (6) et l'Afrique du Nord (3). Par conséquent, il est possible de voir que la proximité géographique des pays européens et la proximité culturelle des pays sud-américains ont favorisé le choix de ces pays comme facteurs de localisation, bien qu'il importe de signaler l'intérêt considérable que suscite le marché américain.

Deuxièmement, à la figure 1, nous illustrons la distribution des IDE selon leurs fonctions. Cela permet de voir que les filiales à caractère exclusivement commercial (avec 67\%) dominent celles qui développent des activités de production. Ce fait s'applique très bien à la petite taille des entreprises étudiées ; beaucoup d'entre elles n'ont qu'un seul IDE. Par conséquent, elles seront sûrement dans les premières étapes de la chaîne d'internationalisation que défend le modèle 
TABLEAU 8

Pays de destination des IDE

\begin{tabular}{lccc}
\hline & Fréquence & Pourcentage & Pourcentage cumulé \\
\hline États-Unis & 10 & 14,5 & 14,5 \\
Allemagne & 8 & 11,6 & 26,1 \\
Portugal & 6 & 8,7 & 34,8 \\
Brésil & 5 & 7,2 & 42,0 \\
Mexique & 5 & 7,2 & 49,3 \\
France & 4 & 5,8 & 55,1 \\
Argentine & 3 & 4,3 & \\
Colombie & 3 & 4,3 & \\
Hong Kong & 3 & 4,3 & \\
Italie & 3 & 4,3 & \\
Royaume-Uni & 3 & 4,3 & \\
Chine & 2 & 2,9 & \\
Maroc & 2 & 2,9 & \\
Russie & 2 & 2,9 & \\
Belgique & 1 & 1,4 & \\
Biélorussie & 1 & 1,4 & \\
Chili & 1 & 1,4 & \\
Émirats arabes unis & 1 & 1,4 & \\
Hollande & 1 & 1,4 & \\
Moldavie & 1 & 1,4 & \\
Pérou & 1 & 1,4 & \\
Roumanie & 1 & 1,4 & \\
Tunisie & 1 & 1,4 & \\
Venezuela & 1 & 1,4 & \\
Total & 69 & 100,0 & \\
\hline
\end{tabular}

d'Uppsala. Comme le signalent Campa et Guillén (1999), l'internalisation des exportations chez les entreprises plus petites constitue en effet le premier pas dans leur processus pour devenir des firmes multinationales.

Une autre idée sous-jacente à l'internationalisation graduelle est que plus tôt on a commencé à exporter, plus on développe de l'expérience et des connaissances, plus on devrait pouvoir progresser dans l'aventure internationale. Par conséquent, nous devrions nous attendre à une relation positive entre le nombre d'années de présence de l'entreprise sur le marché international et le nombre d'IDE qu'elle contrôle. Pour étudier cette association, nous avons vérifié le coefficient de corrélation $r$ de Pearson, qui prend toutefois une valeur négative $(-0,112)$, même s'il n'est pas statistiquement significatif (Sig. =0,543). En conséquence, nous ne pouvons pas affirmer que le nombre d'IDE est en corrélation avec une plus longue expérience internationale. Cependant, ce résultat peut s'expliquer par le fait que la plupart des entreprises ne comptent qu'un seul IDE. 
FIGURE 1

\section{Fonctions des IDE}

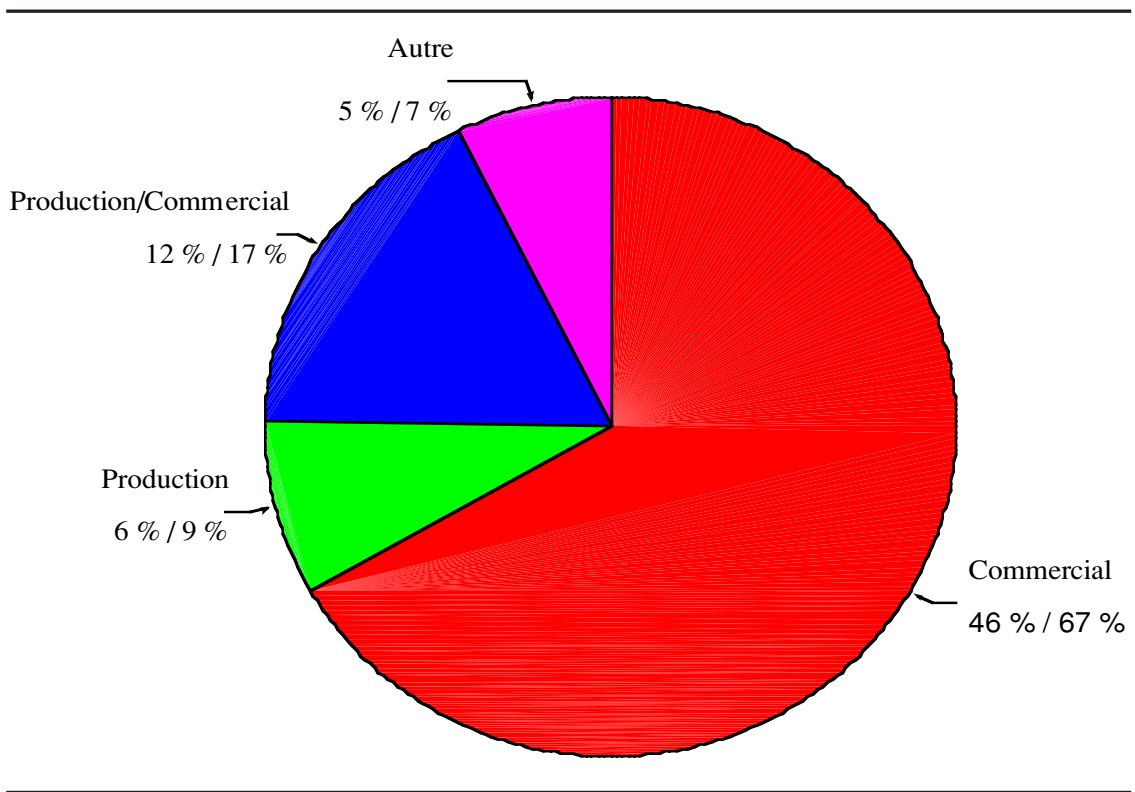

Un autre argument caractérisant ces approches est le cheminement suivi pour s'internationaliser à partir d'une stratégie d'entrée qui comporte moins de risques que l'IDE, pour peu à peu élargir cette stratégie. Dans ce sens, nous n'avons trouvé que trois entreprises qui ont amorcé leur processus d'internationalisation d'une façon différente de l'exportation directe. Au contraire, la plus grande partie des entreprises étudiées ont commencé par de l'exportation (soit 29, c'est-à-dire $90,6 \%)$.

Pour analyser ce gradualisme en suivant une méthode analogue à celle de Cazorla (1996), nous pouvons montrer un modèle séquentiel intégré en cinq types de stratégie d'entrée. Nous pouvons relever le nombre de cas qui ont franchi les 16 étapes possibles en combinant les quatre étapes précédant l'investissement direct, soit l'exportation par un intermédiaire dans le pays d'origine, l'exportation par un intermédiaire dans le pays d'accueil, l'exportation avec du personnel propre envoyé dans le pays d'accueil et des accords contractuels. En considérant chaque cas comme un IDE individuel et non pas comme une entreprise, la distribution de fréquences des étapes franchies avant l'IDE est la suivante. 
FiguRE 2

Séquence suivie pour chaque IDE

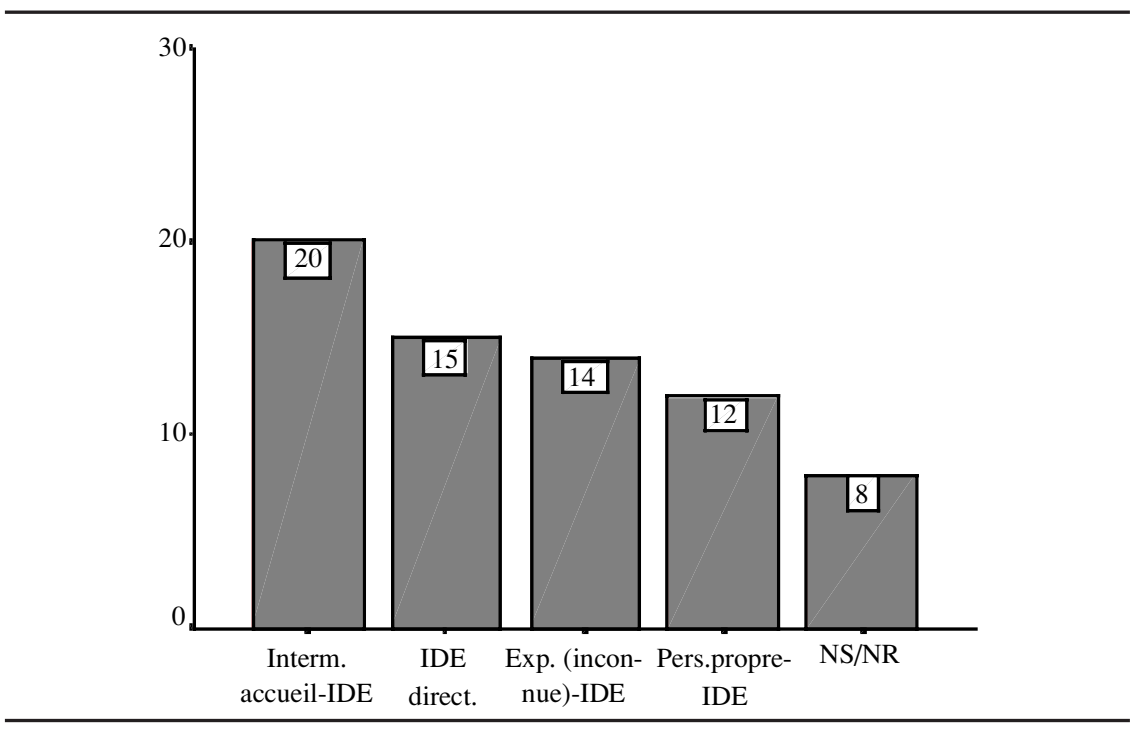

Comme nous pouvons le voir, des 69 IDE détectés, 8 ne connaissaient pas les étapes qui précédaient l'investissement direct à l'étranger. Pour les autres, nous avons relevé quatre séquences : 1) l'exportation par un intermédiaire dans le pays d'accueil, suivi d'IDE, 20 cas ; 2) IDE directement: 15 cas ; 3) exportation sans modalité connue, suivi de l'IDE : 14 cas ; et enfin, 4) exportation avec du personnel propre et l'IDE : 12 cas. En définitive, dans 66,7 \% des cas, une forme d'exportation préalable a été utilisée avant d'investir alors que 21,7\% des IDE ont été effectués sans avoir exporté préalablement dans le pays de destination. Par conséquent, selon les vérifications de Cazorla (1996), nous pouvons observer un cheminement graduel, mais sans passer de façon successive chacune des étapes que prône le modèle d'Uppsala.

Après avoir discuté de l'approche graduelle, nous terminons notre analyse en essayant de mesurer l'influence de la direction sur le processus graduel d'internationalisation et sur les facteurs spécifiques aux PME. Cela relève d'une question à choix multiple dans laquelle on demandait de façon directe si chacun des huit facteurs présentés ci-dessous avait eu une influence significative au moment d'investir, facteurs ou variables dichotomiques prenant une valeur de 1 (si le facteur influençait) ou la valeur de 0 (s'il n'y avait pas d'influence). Le tableau 9 présente la distribution des fréquences ${ }^{17}$.

17. Le cas non répertorié correspond à l'entreprise qui n'a pas décidé d'investir mais qui a repris les installations étrangères d'une entreprise espagnole qu'elle a achetée. 
TABLEAU 9

Facteurs ayant influencé l'IDE

\begin{tabular}{lcc}
\hline Variable & Chiffre & \% de cas (31 cas = 100 \%) \\
\hline Influence de la mentalité de la direction & 23 & 74,2 \\
Influence du processus graduel & 21 & 67,7 \\
Influence de l'activité conjointe & 14 & 45,2 \\
Influence du soutien public & 11 & 35,5 \\
Influence de la destination dans les pays & & 32,3 \\
en voie de développement & 10 & 22,6 \\
Influence des entreprises espagnoles établies & 7 & 19,4 \\
Influence du fait de suivre le client & 6 & 9,7 \\
Influence des importations préalables & 3 & I cas perdu $; 31$ cas valides \\
\hline
\end{tabular}

Comme nous pouvons le constater, les facteurs les plus valorisés sont au nombre de 3 (nous indiquons le pourcentage d'entreprises que chacun a signalé), soit: une ouverture internationale de la direction $(74,2 \%)$, suivi du recours à un processus graduel $(67,7 \%)$ et d'activités conjointes $(45,2 \%)$. Quant à la coopération entre entreprises pour réaliser de l'IDE, il est possible de trouver une donnée supplémentaire, en vertu de la distribution de fréquences illustrée à la figure 3 : les filiales de pleine propriété (avec $57 \%$ des observations) et les coentreprises (avec $26 \%$ ) sont les deux principaux modes d'établissement utilisés par les entreprises enquêtées. Par conséquent, en dépit de l'importance du poids relatif que présentent les investissements conjoints, le fait qu'il s'agisse de PME ne semble pas empêcher que ces investissements s'effectuent en solitaire.

Finalement, le fait de considérer un pays moins développé comme destination peut avoir joué un rôle dans certaines décisions d'investissement en Amérique latine ou en Afrique du Nord, même si ces options ont été peu valorisées, car, comme on peut le voir au tableau 8, ces deux zones reçoivent un peu plus de $30 \%$ des IDE.

\section{Conclusion}

Les éléments présentés dans cet article visaient à éclairer un phénomène qui a traditionnellement été considéré comme relevant exclusivement des grandes entreprises, soit la possibilité d'investir directement à l'étranger. Ainsi, à partir de l'ensemble des entreprises analysées, nous avons pu établir le profil type de la PME engagée dans l'IDE. Il s'agit fondamentalement d'une entreprise comptant moins de 150 employés, qui a un chiffre d'affaires annuel d'environ 19,7 millions d'euros, qui appartient à l'un des secteurs de plus forte expérience internationale

Revue internationale P.M.E., vol. 16, $\mathrm{n}^{\circ} 1,2003$ 
FIGURE 3

Modalités d'IDE

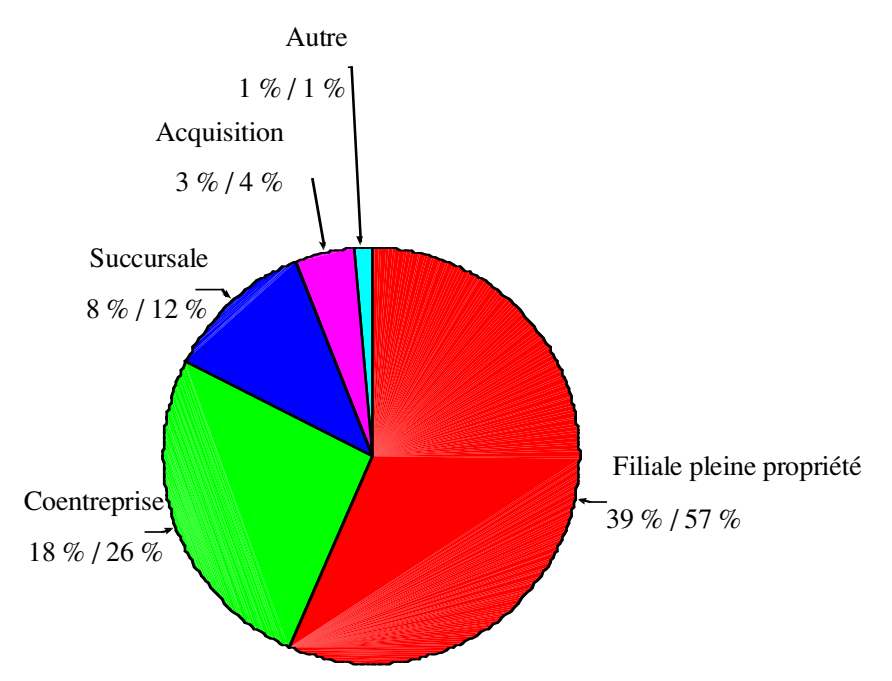

dans son territoire d'origine (dans notre cas, la province d'Alicante), qui a amorcé son parcours international il y a déjà vingt ans et qui exporte pour environ 10,5 millions d'euros par an, somme qui correspond à un peu plus de la moitié du chiffre d'affaires.

Après cette analyse descriptive, nous avons voulu vérifier le paradigme éclectique dans la décision des entreprises d'investir à l'étranger. Les responsables de l'international interviewés accordent une grande importance d'une part aux avantages que possède leur entreprise par rapport aux concurrents nationaux et, d'autre part, à la qualité de l'organisation pour gérer les transactions. Ensuite, dans l'exposé théorique, nous avons insisté sur une série de facteurs qui, précisément, montrent que la PME peut se compromettre avec l'IDE. Finalement, les facteurs les plus influents sont l'existence d'une forte ouverture internationale de la direction, la mise en place d'un processus graduel enclenché par des stratégies moins ambitieuses et l'internationalisation conjointe avec d'autres entreprises.

L'internationalisation graduelle a été l'objet d'une attention spéciale. Rappelons en premier que la plupart des PME étudiées ont un seul IDE, ce qui en fait des «multinationales naissantes ». En second lieu, une grande partie des IDE est dirigée vers l'Amérique latine, soulignant l'importance d'une proximité psychique qui se combine avec une distance physique plus faible; de même, les IDE localisés dans les pays européens sont plus nombreux. Aussi, en accord avec l'approche étapiste, nous pouvons signaler que presque toutes les entreprises ont 
démarré leur processus d'internationalisation par des exportations et que les IDE sont souvent précédés de stratégies d'entrée moins risquées dans le pays en question. En outre, la plupart des filiales étrangères ont un caractère exclusivement commercial, ce qui permet de situer ces PME dans les premières étapes de la séquence d'internationalisation du modèle d'Uppsala. Cependant, nous n'avons pas pu confirmer l'importance d'une plus grande ancienneté internationale pour justifier un plus grand nombre d'IDE.

Ces résultats empiriques devraient être complétés par des analyses visant d'autres territoires géographiques et des études approfondies, comme celles recourant à l'étude de cas. Il serait ainsi possible de vérifier tous les autres éléments soulevés par les théories expliquant l'IDE ainsi que d'autres facteurs plus spécifiques, concernant par exemple la naissance et le développement du phénomène des PME multinationales.

En guise de conclusion, nous aimerions faire ressortir les implications conceptuelles et pratiques de notre travail. Sur le plan théorique, nous considérons, comme principal apport, l'intégration de différents facteurs dans un même cadre conceptuel en ce qui concerne la décision de la PME d'investir à l'étranger. Sur le plan pratique, notre recherche peut contribuer à la diffusion auprès des responsables internationaux dans les PME des ressources sur lesquelles ils peuvent compter pour amener leur entreprise à franchir des étapes plus avancées du processus d'internationalisation.

\section{Bibliographie}

Alonso, J.A. (1993), "Capacidades exportadoras y estrategia internacional de la empresa », dans J. Velarde, J.L. García Delgado et A. Pedreño (dir.), Empresas y empresarios españoles en la encrucijada de los noventa, Madrid, Ed. Civitas, p. 205-258.

AlONSO, J.A. (1994), «El proceso de internacionalización de la empresa », Información Comercial Española, $\mathrm{n}^{\mathrm{0}}$ 725, p. 127-143.

Alonso, J.A. et V. Donoso (1996), «Obstáculos a la internacionalización y políticas públicas de promoción. El caso de España», Papeles de Economía Española, $\mathrm{n}^{\mathrm{o}} 66$, p. $124-143$.

ANDERSEN, O. (1993), « On the internationalization process of firms : a critical analysis », Journal of International Business Studies, $2^{\mathrm{e}}$ trimestre, p. 209-231.

ARMSTRONG, J.S. et T.S. OVERTON (1977), «Estimating nonresponse bias in mail surveys », Journal of Marketing Research, vol. XIV, août, p. 396-402.

ARNOLD, D.J. et J.A. QUELCH (1998), «New strategies in emerging markets », Sloan Management Review, automne, p. 7-20.

BAYAD, M. (1997), «Internationalisation et pratiques de gestion des ressources humaines en PME », Revue internationale PME, vol. 10, $\mathrm{n}^{\text {os }} 3-4$, p. 57-80.

Revue internationale P.M.E., vol. 16, nº 1, 2003 
BILKEY, W.J. et G. TESAR (1977), «The export behavior of smaller-sized Wisconsin manufacturing firms », Journal of International Business Studies, vol. 8, n 1, p. 93-98.

BUCKLEY, P.J. (1989), « Foreign direct investment by small and medium sized enterprises : the theoretical background », Small Business Economics, vol. 1, p. 89-100.

BuCKLEY, P.J. et M. CASSON (1976), The Future of the Multinational Enterprise,

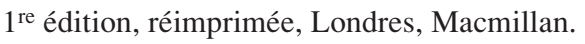

Calvo, F. (1997), Internacionalización. Un reto para la empresa alicantina, Fundesem y Cámara Oficial de Comercio, Alicante, Industria y Navegación de Alicante.

CAMISón, C. (1997), «Inversión directa comercial en el exterior a partir de acuerdos de cooperación entre PyMEs españolas : el caso de los proyectos cofinanciados por el ICEX », VII Congreso Nacional de ACEDE, p. 345-360.

CAMPA, J.M. et M.F. GuILlÉN (1999), «The internalization of exports : firm - and location - specific factors in a middle-income country », Management Science, vol. $45, \mathrm{n}^{\circ} 11$, p. 1463-1478.

CAVusgiL, S.T. (1980), «On the internationalization process of firms », European Research, novembre, p. 273-281.

CAzORLA, L. (1996), «La inversión directa en países en vía de desarrollo : una estrategia al alcance de la pequeña y mediana empresa industrial española», VI Congreso Nacional de ACEDE, p. 84-99.

CAzORLA, L. (1997), «La inversión directa de la PyME industrial española en países en vías de desarrollo: un análisis de los factores determinantes », Información Comercial Española, no 761, p. 49-66.

ChetTy, S. et D. Blankenburg Holm (2000), «Internationalisation of small to mediumsized manufacturing firms : a network approach », International Business Review, vol. 9, no 1, p. 77-93.

CONSEIL SUPÉRIEUR DE CHAMBRES DE COMMERCE, INDUSTRIE ET DE NAVIGATION (1990), Acceso de la empresa a los mercados exteriores. Cooperación al desarrollo. "Facilidades Cheysson », Madrid, p. 1.

Coviello, N.E. et A. MCAuley (1999), «Internationalisation and the smaller firm : a review of contemporary empirical research », Management International Review, vol. 3, no 3, p. 223-256.

Czinkota, M.R. (1982), Export Development Strategies. US Promotion Policy, New York, Praeger.

DARRÉON, J.L. et S. FAIÇAL (1993), «Les enjeux des partenariats stratégiques entre grandes entreprises et PME », Revue française de gestion, septembre-octobre, p. 104-115.

DONCKELS, R. et J. LAMBRECHT (1995), «La coentreprise comme lien de collaboration entre les PME des pays développés et en voie de développement », Revue internationale PME, vol. 8, no 1, p. 9-29.

DUNNING, J.H. (1979), «Explaining changing patterns of international production: in defense of the eclectic theory », Oxford Bulletin of Economics and Statistics, vol. 41, p. 269-295.

DunNING, J.H. (1981), International Production and the Multinational Enterprise, Londres, George Allen \& Unwin, p. 49 et 80-111.

Revue internationale P.M.E., vol. 16, $\mathrm{n}^{\circ}$ 1, 2003 
DUNNING, J.H. (1988), «The eclectic paradigm of international production : a restatement and some possible extensions », Journal of International Business Studies, vol. 19, p. 1-32.

Eriksson, K., J. Johanson, A. Majkgard et D.D. Sharma (1997), «Experiental knowledge and cost in the internationalization process », Journal of International Business Studies, $2^{\mathrm{e}}$ trimestre, p. 337-360.

GIBIAT, M. (1994), «Les modèles intégrés de la décision d'exporter en PME / PMI : synthèse des recherches depuis 20 ans », Revue internationale PME, vol. 7, n 2, p. 11-29.

GIDDY, I. H. et S. Young (1982), «Conventional theory and unconventional multinationals : do new forms of multinational enterprise require new theories?» dans A.M. Rugman (dir.), New Theories of the Multinational Enterprise, Londres, Croom Helm, p. 55-78.

HÉGO, E. (1994), «Le recours des PME au conseil à l'export, une démarche réelle mais encore confuse : le cas des industries agro-alimentaires », Revue internationale PME, vol. 7, n $\mathrm{n}^{\mathrm{O}}$ 2, p. 137-158.

HITT, M.A. et B.R. BARTKUS (1997), «International entrepreneurship », dans J.A. Katz (dir.), Advances in Entrepreneurship, Firm Emergence and Growth, Greenwich, Jai Press, p. 7-30.

HYMER, S.H. (1976), The International Operations of National Firms : A Study of Direct Foreign Investment, Cambridge, Massachusetts, The MIT Press.

IBORRA, M. et M. RIPOLLÉS (1997), «Redes empresariales y nuevas empresas internacionales : ¿un reto al enfoque gradualista de la internacionalización? », VII Congreso Nacional de ACEDE, p. 388-403.

JOHANSON, J. et L.G. MATTSSON (1988), «Internationalisation in industrial systems - a network approach», dans N. Hood et J.E. Vahlne (dir.), Strategies in Global Competition, Londres, Croom Helm, p. 287-314.

JOHANSON, J. et J.E. VAHLNE (1977), «The internationalization process of the firm. A model of knowledge development and increasing foreign market commitments », Journal of International Business Studies, vol. 8, nº 1, p. 23-32.

JOHANSON, J. et J.E. VAHLNE (1990), «The mechanism of internationalization », International Marketing Review, vol. 7, no 4, p. 11-24.

JOHANSON, J. et F. WIEDERSHEIM-PAUL (1975), «The internationalization of the firm. Four Swedish cases », The Journal of Management Studies, octobre, p. 305-322.

KInDleBerger, C.P. (1969), American Business Abroad: Six Lectures on Direct Investment, New Haven, Connecticut, Yale University Press.

Korhonen, H., R. LuOSTARINEN et L. WELCH (1996), «Internationalization of SME's : inward-outward patterns and government policy », Management International Review, vol. 36, no 4, p. 315-329.

LÉO, P.Y. (2000), «Les PMI et les couples produits-services à l'exportation », Revue internationale PME, vol. 13, n 2, p. 45-73.

MAhone, C.E. et P.K. Choudhury (1995), « Small and medium sized manufactures and traders », Multinational Business Review, automne, p. 17-26.

Revue internationale P.M.E., vol. 16, n 1, 2003 
MARTínez MorA, C. (1997), Causas de internacionalización de las empresas : un estudio aplicado a pequeñas y medianas empresas de la provincia de Alicante, Alicante, Instituto de Cultura Juan Gil-Albert.

McDougall, P.P., S. ShanE et B.M. OviatT (1994), «Explaining the formation of international new ventures: the limits of theories from international business research », Journal of Business Venturing, no 9, p. 469-487.

MiESENBOCK, K.J. (1988), « Small business and exporting : a literature review », International Small Business Journal, vol. 6, n 2, p. 42-61.

Oviatt, B.M. et P.P. MCDougall (1994), «Toward a theory of international new ventures », Journal of International Business Studies, $1^{\mathrm{er}}$ trimestre, p. 45-64.

PAN, Y. et D.K. TSE (1996), « Cooperative strategies between foreign firms in an overseas country », Journal of International Business Studies, numéro spécial, p. 929-945.

PLA, J. et S.M. SUÁREZ (2000), « ¿Cómo se explica la internacionalización de la empresa? Una perspectiva teórica integradora », XIV Congreso Nacional de AEDEM, p. 1-19.

Planellas, M. (1997), «El network del creador de empresa », Iniciativa Emprendedora y Empresa Familiar, nำ 7, p. 11-15.

REID, S.D. (1981), «The decision-maker and export entry and expansion », Journal of International Business Studies, automne, p. 101-112.

REUBER, A.R. et E. FISCHER (1997), « The influence of the management team's international experience on the internationalization behaviors of SMEs », Journal of International Business Studies, 4e trimestre, p. 807-825.

RiAlP, A. et J. RialP (1996), «El papel de los acuerdos de cooperación en los procesos de internacionalización de la empresa española : un análisis empírico », Papeles de Economía Española, no 66, p. 248-266.

Root, F.R. (1982), Foreign Market Entry Strategies, New York, AMACOM.

Rugman, A.M. (1981), Inside the Multinationals, Grande-Bretagne, New York Columbia University Press.

SuÁREZ, S., J.M. GARCía et R. ÁlAmo (1999), «El compromiso exportador de las empresas: un modelo basado en las percepciones directivas », Primer Congreso Internacional Iberoamerican Academy of Management, p. 1-29.

VAlenzuela, A. (2000), «Modelo de capacidades para la internacionalización de la empresa exportadora española», Cuadernos de Economía y Dirección de la Empresa, no 5, p. 91-109.

VeCiAnA, J.M. et E. GENESCÁ (1994), «Management y competitividad en la empresa española », Alta Dirección, no 174 , p. 21-36.

VERNON, R. (1966), «International investment and international trade in the product cycle », Quarterly Journal of Economics, vol. 80, p. 190-207.

VERNON, R. (1973), Soberanía en peligro. La difusión multinacional de las empresas de Estados Unidos, México, Fondo de Cultura Económica.

VERNON, R. (1979), « The product cycle hypothesis in a new international environment », Oxford Bulletin of Economics and Statistics, vol. 41, no 4, novembre, p. 255-267.

WELLS, L.T. (1968), «A product life cycle for international trade ?» Journal of Marketing, vol. 32, p. 1-6.

Revue internationale P.M.E., vol. 16, nº 1, 2003 